Science and Technology

Elsevier Editorial System(tm) for Composites

Manuscript Draft

Manuscript Number: CSTE-D-07-00396R1

Title: Determination of fiber length in axially symmetrical metal matrix composites

Article Type: Full Length Article

Section/Category:

Keywords: A. Metal-matrix composites; A. Short-fiber composites; B.

Microstructure; E. Extrusion; E. Powder processing.

Corresponding Author: Dr Gaspar González-Doncel, PhD

Corresponding Author's Institution: CENIM, C.S.I.C.

First Author: Alberto Borrego, PhD

Order of Authors: Alberto Borrego, PhD; Joaquín Ibáñez, PhD; Gaspar GonzálezDoncel, PhD

Manuscript Region of Origin:

Abstract: A procedure to calculate the spatial fiber length in discontinuously reinforced composites from data of conventional metallographical images is developed. The method is based on the following three assumptions: first, the measured length of a reinforcing fiber in a metallographical picture is a projection of the actual fiber length; second, the alignment of short fibers is axially symmetrical (for example, extruded composites in which the extrusion direction is a symmetry axis for the orientation of reinforcing fibers); third, for a representative number of fibers, the dependence of the projected length with the corresponding projected angle, along a given sample direction, is known. Either analytical or numerical solution can be found depending on the specific dependence of the projected length with the projected angle. This procedure has been applied to real 6061Al-15volosiCw composites obtained by a powder metallurgical route in which the final step for materials consolidation was extrusion of powder blends at different temperatures. 


\title{
Determination of fiber length in axially symmetrical metal matrix composites
}

\author{
Alberto Borrego, Joaquín Ibáñez, Gaspar González-Doncel \\ Dept. of Physical Metallurgy, Centro Nacional de Investigaciones Metalúrgicas (CENIM), C.S.I.C. \\ Avda. de Gregorio del Amo, 8, 28040 Madrid, Spain
}

\begin{abstract}
A procedure to calculate the spatial fiber length in discontinuously reinforced composites from data of conventional metallographical images has been developed. The method is based on the following three assumptions: first, the measured length of a reinforcing fiber in a metallographical picture is a projection of the actual fiber length; second, the alignment of short fibers is axially symmetrical (for example, extruded composites in which the extrusion direction is a symmetry axis for the orientation of reinforcing fibers); third, for a representative number of fibers, the dependence of the projected length with the corresponding projected angle, along a given sample direction, is known. Either analytical or numerical solution can be found depending on the specific dependence of the projected length with the projected angle. This procedure has been applied to real $6061 \mathrm{Al}-15 \mathrm{vol} \% \mathrm{SiC}_{\mathrm{w}}$ composites obtained by a powder metallurgical route in which the final step for materials consolidation was extrusion of powder blends at different temperatures.
\end{abstract}

Key words: Short Fiber Reinforcement, Quantitative Metallography, Axial Symmetry, Metal Matrix Composites, Aluminum alloys.

* Corresponding author: Tel.:+34-915538900, ext.337, fax: +34-915347425,

E-mail: ggd@cenim.csic.es 


\section{Introduction}

For predicting the mechanical properties of discontinuously reinforced metal matrix composites with short fibers; e.g., $\mathrm{Al}-\mathrm{SiC}_{\mathrm{w}}$, it is generally agreed that a very important mechanism in explaining the improved response of these materials (higher stiffness, yield stress, work hardening rate and strength) is the transfer of load from matrix to the rigid reinforcement (1-3). This mechanism is usually described either through Eshelby or ShearLag models (4-11). In both cases, data of some microstructural parameters related to the reinforcement phase are required if reliable predictions of the mechanical properties are to be sought. These parameters include, volume fraction, aspect ratio, $l / d$ (with $l$ and $d$ length and diameter of the fiber, respectively), and degree of alignment of the fibers with the applied load direction. Also, the degree of homogeneity of the reinforcement in the matrix plays an important role in the mechanical response of these composites $(12,13)$, but due to the intrinsic complexity of the mathematical treatment, it will not be treated here and, rather well homogeneous composites will be considered.

Whereas the volume fraction does not depend on the fabrication procedure and is usually known from material specification, the aspect ratio of the short fibers and their degree of alignment are strongly dependent on the specific fabrication procedure and on the parameters involved in material preparation (14). For this reason, the mechanical properties of these composites are highly sensitive to the preparation procedures (15-17). Knowledge of the above two parameters requires, therefore, detailed measurements of a representative number of individual fibers. This operation is usually carried out directly from conventional observations in the optical or scanning electron microscope, SEM.

It is usually asumed that the materials have perfectly aligned fibers when load transfer is calculated by either of the above two kind of models (Eshelby and Shear-Lag). This is because; first, it is difficult (or even unattainable) to obtain a complete and reliable description of the fiber alignment from conventional optical or scanning electron microscopy, 2D, and, second, it highly simplifies the mathematical treatment of the problem. The microstructure of real, 3D, composite materials is, however, far more complex. Typically, an unknown orientation distribution function, rather than perfectly aligned fibers (Dirac's delta function), gives a proper description of fiber alignment. That is to say, the reinforcement is not perfectly aligned but misaligned to a certain degree, with a specific sample direction. 
The results obtained from a two dimensional, 2D, description are very close to the 3D function in the case of well aligned fibers if the appropriate metallographical section is prepared. However, they become increasingly unreliable as the misalignment rises, since projections of the fiber length and misorientation angle are actually being measured.

It is, therefore, of prime interest to develop a procedure which provides a $3 \mathrm{D}$ microstructural description of discontinuously reinforced composites in the light of common 2D metallographical observations. This is the purpose of the present investigation. The procedure described here will be applied to materials with an axial symmetry of the reinforcement orientation for which either an analytical or numerical solution can be obtained. This is the case when composites preparation involves extrusion into bars. Due to the axial symmetry imposed by this deformation process, fiber alignment achieves an axi-symmetrical 3D distribution in which the extrusion direction is the symmetry axis. The method described will be applied to some real $6061 \mathrm{Al}-15 \mathrm{vol} \% \mathrm{SiC}_{\mathrm{w}}$ composites obtained by a powder metallurgical route in which the final step for materials preparation was extrusion of powder blends at different temperatures.

\section{Analytical procedure}

Let us assume a metallographical section prepared on a surface containing the symmetrical axis of the sample. For a metallographic preparation in which fiber length is not modified (e.g. by polishing with diamond paste and/or silicon colloidal on a soft polishing cloth), individual fibers must jut out from the metallic matrix surface (metallographical plane) (18, 19). The main parameters that can be measured are: the projected length, $l^{\prime}$, of the fiber and the angle, $\theta^{\prime}$, of the fiber with the symmetry axis, as shown in the scheme of Figure 1 . This figure also shows the azimuth angle, $\beta$. From elementary trigonometry we have,

$$
\begin{aligned}
& l=l^{\prime} \frac{\cos \theta^{\prime}}{\cos \theta} \\
& \operatorname{tg} \theta^{\prime}=\cos \beta \cdot \operatorname{tg} \theta \\
& \cos \theta^{\prime}=\frac{1}{\left(1+\operatorname{tg}^{2} \theta^{\prime}\right)^{1 / 2}}
\end{aligned}
$$

where, $\pi / 2 \geq \beta \geq \theta^{\prime} \geq 0$. 
From equations $[1 \mathrm{~b}]$ and $[1 \mathrm{c}]$,

$$
\cos \theta^{\prime}=\frac{1}{\left(1+\cos ^{2} \beta \cdot \operatorname{tg}^{2} \theta\right)^{1 / 2}}
$$

Replacing [2] into [1a], it is possible to get the dependence of the spatial length, $l$, with the projected length, $l^{\prime}$, in terms of $\theta$ and $\beta$ :

$$
l=l^{\prime} \cdot\left[\cos \theta \cdot\left(1+\cos ^{2} \beta \cdot \operatorname{tg}^{2} \theta\right)^{1 / 2}\right]^{-1}
$$

It can be seen from this equation, as well as directly from Figure 1, that both, $\theta$ and $\beta$, besides the projected length $l^{\prime}$, are needed to calculate $l$ for any given fiber. If a large number of fibers are measured, a representative value of $l^{\prime}$ as a function of the angle $\theta^{\prime}$ is obtained. Then, $l$ and $l^{\prime}$ in equation [3] can be replaced by $l^{\prime}\left(\theta^{\prime}\right)$ and $l(\theta, \beta)$, respectively. So, the following correlation between values in three dimension $(l, \theta$ and $\beta)$ and their projections in two dimensions $\left(l^{\prime}, \theta^{\prime}\right)$ is derived from equation [3].

$$
l^{\prime}\left(\theta^{\prime}\right)=l(\theta, \beta) \cdot L(\theta, \beta)
$$

where

$$
L(\theta, \beta)=\left[\cos \theta \cdot\left(1+\cos ^{2} \beta \cdot \operatorname{tg}^{2} \theta\right)^{1 / 2}\right]
$$

From equation [4], and using equation [1b], the following relation can be obtained,

$$
l(\theta, \beta)=l^{\prime}\left(\theta^{\prime}\right) \cdot[L(\beta, \theta)]^{-1}=l^{\prime}\left(\operatorname{tg}^{-1}(\cos \beta \cdot \operatorname{tg} \theta)\right) \cdot\left[\cos \theta \cdot\left(1+\cos ^{2} \beta \cdot \operatorname{tg}^{2} \theta\right)^{1 / 2}\right]^{-1}
$$

Equation [5] describes the dependence of the spatial length function on $\theta$ and $\beta$, i.e. $l(\theta, \beta)$, using $l^{\prime}\left(\theta^{\prime}\right)$, which is the measurable data for a representative number of fibers obtained through metallographical sections.

In order to find a solution for equation [5] further hypotheses on $\beta$ are required. This is because it is not possible to calculate the three dimensional length in terms of only the two dimensional projection of the above magnitudes. From a metallographical section it is possible to determine $l^{\prime}$ and $\theta^{\prime}$, but not $\theta$ and $\beta$.

The fact that a large number of short fibers is taken into account for the analysis of the spatial length distribution allows us to select the necessary hypothesis to obtain $l(\theta, \beta)$. In axially 
symmetrical materials, each fiber may lie at a given angle $\theta$ with the extrusion axis, but its length, $l$, does not depend on the azimuth angle because of the symmetry around the extrusion axis. Therefore, the average value of $l$ over $\beta$ for a given $\theta, \bar{l}(\theta)$, can be obtained by simple integration of equation [5].

$$
\bar{l}(\theta)=\int_{0}^{\pi / 2} \frac{l^{\prime}\left[\operatorname{tg}^{-1}(\cos \beta \cdot \operatorname{tg} \theta)\right]}{L(\theta, \beta)} d \beta=\frac{1}{\pi / 2 \cos \theta} \int_{0}^{\pi / 2} \frac{l^{\prime}\left[\operatorname{tg}^{-1}(\cos \beta \cdot \operatorname{tg} \theta)\right]}{\left(1+\cos ^{2} \beta \cdot \operatorname{tg}^{2} \theta\right)^{1 / 2}} d \beta
$$

For materials in which an analytical expression for $l^{\prime}\left(\theta^{\prime}\right)$ cannot be obtained or the resulting integral cannot be solved by a straightforward way, it is always possible to reach a solution of equation [6] by numerical integration. In the simplest case in which $l^{\prime}\left(\theta^{\prime}\right)$ is a constant, $l^{\prime}\left(\theta^{\prime}\right)=\mathrm{R}$, the integral of equation [6] can be reduced to a first class complete elliptical integral $F(\sin \theta, \pi / 2)($ see appendix):

$$
\bar{l}(\theta)=\frac{2 R}{\pi} \int_{0}^{\pi / 2} \frac{d \beta}{\left(1-\sin ^{2} \theta \cdot \sin ^{2} \beta\right)^{1 / 2}}=\frac{2 R}{\pi} F(\sin \theta, \pi / 2)
$$

The values for $F(\sin \theta, \pi / 2)$ are tabulated and can be obtained from an applied mathematics handbook (20).

Figure 2 shows the dependence of the average length of the short fibers, in arbitrary units, with the misorientation angle, $\bar{l}(\theta)$, for the case in which $l^{\prime}\left(\theta^{\prime}\right)=\mathrm{R}$; i.e., constant projected length ( $\mathrm{R}=1$ in arbitrary units). As can be seen, at low angles, both projected and actual lengths are very similar, but they strongly diverge with increasing the misorientation angle.

\section{Application to real composites and discussion}

In previous work, the influence of extrusion temperature, $\mathrm{T}_{\mathrm{ext}}$, on the microstructure and the texture of $6061 \mathrm{Al}-15 \mathrm{vol} \% \mathrm{SiC}_{\mathrm{w}}$ composites was investigated (14). It was seen that the extrusion process results in the alignment of a number of fibers with the extrusion flow direction, most of them remaining aligned at random. The extrusion temperature, $\mathrm{T}_{\text {ext }}$, mostly influenced the degree of whisker orientation, which follows a Gaussian distribution function, $\mathrm{I}(\theta)=a \exp \left(-b \theta^{2}\right)$, where $a$ and $b$ are constants, specifically of the SiC whiskers distribution in each composite. The extrusion temperature, however, had little effect on the relative amount of oriented versus misoriented whiskers. 
Table 1 summarizes $T_{\text {ext }}$, the volume fraction of whiskers which are aligned with the extrusion axis, $F_{v}$ (the remaining fraction, $1-F_{v}$, refers to whiskers randomly oriented in the aluminum matrix or equiaxed $\mathrm{SiC}$ particles), and the parameters $a$ and $b$ of the Gaussian distribution function. The trend of $\mathrm{SiC}$ whiskers to be aligned with the extrusion axis can be seen in Figure 3. This figure, an inverse stereographic triangle for the extrusion axis (inverse pole figure), corresponds to the $\mathrm{SiC}$ phase of the composite material extruded at $498^{\circ} \mathrm{C}$. It was obtained by x-ray diffraction texture analysis as described in (14). Since the SiC whiskers are single crystals in which the crystallographic $<111>$ is parallel to the long direction of the fiber, the intensity of the contours levels of such an inverse pole figure is directly related to the relative number of $\mathrm{SiC}$ whiskers misaligned at a given angle $\theta$ with the extrusion axis. As can be seen from Figure 3, a maximum is obtained on the $<111>$ corner of the stereographic triangle showing the trend of the $\mathrm{SiC}$ short fibers to be aligned with the extrusion axis. Figure 3 also shows the axial symmetry of the oriented population of $\mathrm{SiC}$ whiskers around the extrusion axis.

For the purpose of measuring $l^{\prime}$, and $\theta^{\prime}$ of individual short fibers in these four composites, a representative number of $\mathrm{SiC}$ whiskers have been analyzed in each material by means of quantitative metallography following a procedure similar to that used by Morimoto et al (18). Measurements were conducted on SEM images at X3000 magnification. It is assumed here, however, that part of the whisker is embedded in the matrix. On average, it can be assumed that $1 / 2$ of the total length of the fiber juts out from the surface if no fiber breakage occurs during metallographical preparation. Figure 4 shows a longitudinal SEM micrograph of the composite extruded at $498^{\circ} \mathrm{C}$ and the processed image used to carry out the measurements of $l^{\prime}$, and $\theta^{\prime}$. Data was obtained using appropriate image analyzer software. A representative number of whiskers $(\approx 600)$, with a minimum aspect ratio of about 1.5 , were measured in each composite.

Data of $l^{\prime}$ are shown in the plots of Figure 5. This figure presents average values of $l^{\prime}$ for each material as a function of $\theta^{\prime}$. As can be seen, $l^{\prime}$ is barely affected by $\mathrm{T}_{\text {extr }}$ which leads to an average projected whisker length of about $1.75 \mu \mathrm{m}$ for all composites (projected aspect ratio about 4). It can also be seen that there is little variation of $l^{\prime}$ with $\theta^{\prime}$ in the materials extruded at low temperature, $\mathrm{C} 32$ and $\mathrm{C} 34$, whereas there is a slight tendency to decrease $l^{\prime}$ with the increase in $\theta^{\prime}$ as extrusion temperature is raised, C38 and C45. 
Since the composite microstructure has axial symmetry around the extrusion axis, the actual length, $l$, does not depend on the azimuth angle, $\beta$, and its dependence with misorientation angle, $\theta$, can be obtained from equation [7].

The results from numerical integration of equation [7] for the four composites investigated are shown in Figure 6. It is observed that fiber length, $l$, increases with misorientation angle $\theta$, in all materials, and does not depend on the extrusion temperature, showing that the orientation process of short $\mathrm{SiC}$ fibers during composite extrusion is a source of fiber breakage. As stated in a previous work (14), plastic flow during extrusion at the microscopic scale occurs in a rather turbulent manner, and results in a Gaussian orientation distribution function for oriented fibers. Hence, the larger the rotation a whisker should undergo, the higher the probability of whisker breakage during rotation.

It is finally worth mentioning the importance of careful sample metallographical preparation for reliable measurements. In other words, breakage of jutted out fibers should be avoided during sample preparation for a reliable three dimensional measurement.

\section{Summary}

In this work, a method to calculate the actual fiber length from data of two dimensional metallographical images in discontinuously reinforced composites has been developed. The following three assumptions should be made:

1.- The measured length of any given individual fiber is a projection on the micrograph plane of the actual length of the reinforcing fiber.

2.- Alignment of short fibers is axially symmetrical. Such is the case for extruded composites into cylindrical bars in which the extrusion direction is a symmetry axis for the orientation of the reinforcing fibers.

3.- The dependence of projected length with projected angle on a given sample direction for a representative number of fibers is known, either in analytical or numerical form.

The method has been applied to real composite materials obtained by powder metallurgy involving axially symmetrical extrusion as the final step in their preparation. The results show that the fiber rotation during the extrusion process is a source of fiber breakage in the course of composite fabrication. 


\section{Acknowledgement}

Projects MAT01-2085 from MCYT and MAT05-0527 from MEC, Spain.

\section{References}

1. Kelly A, Davies GJ. The principles of the fibre reinforcement of metals. Metall.Rev. 1965;10: 1-77.

2. Miller WS, Humphreys FJ, Strengthening mechanisms in particulate metal matrix composites, Scr.Metall. 1991; 25: 33-38.

3. Christman T, Needleman A, Suresh S. An experimental and numerical study of deformation in metal-ceramic composites. Acta Metall. 1989; 37: 3029-3050.

4. Withers PJ, Stobbs WM, Pedersen OB. The application of the Eshelby method of internal stress determination to short fibre metal matrix composites. Acta Metall. 1989; 37: 30613084.

5. Taya M, Lulay KE, Lloyd DJ. Strengthening of a particulale metal matrix composite by quenching. Acta Metall.Mater. 1991; 39: 73-87.

6. Arsenault RJ, Taya M. Thermal residual stress in metal matrix composite, Acta Metall. 1987; 35: 651-659.

7. Nardone VC, Strife JR. Analysis of the creep behavior of silicon carbide whisker reinforced 2124Al (T4). Metall Trans 1987; 18A: 109-114.

8. Nardone VC, Prewo KM. On the strength of discontinuous silicon carbide reinforced aluminum composites. Scr.Metall. 1986; 20: 43-48.

9. Nairn JA. On the use of shear-lag methods for analysis of stress transfer in unidirectional composites. Mech.Mater. 1997; 26: 63-80.

10. Llorca J. An analysis of the influence of reinforcement fracture on the strength of discontinuously reinforced metal matrix composites. Acta Metall.Mater. 1995; 43: 181192.

11. Ganguly P, Poole WJ. Characterization of reinforcement distribution inhomogeneity in metal matrix composites. Mater Sci Eng 2002; 332A: 301-310.

12. Ryu Ho, Chung K-H, Cha S-I, Hong S-H. Analysis of creep ehavior of SiC/Al metal matrix composites based on a generalized shear-lag model. J.Mater.Res. 2004; 19: 36333640 .

13. Segurado J, González C, Llorca J. A numerical investigation of the effect of particle clustering on the mechanical properties of composites. Acta Mater. 2003; 51: 2355-2369.

14. Borrego A, Fernández R,Cristina MC, Ibáñez J, González-Doncel G; Influence of extrusion temperature on the microstructure and the texture of $6061 \mathrm{Al}-15 \mathrm{vol} \% \mathrm{SiCw} \mathrm{PM}$ composites. Comp.Sci.Tec. 2002; 62: 731-742.

15. M.Kouzeli M, Mortensen A. Size dependent strengthening in particle reinforced aluminum. Acta Metall.Mater. 2002; 50: 39-51. 
16. D.Srinivasan D, Surappa MK. Effect of iron impurity and thermomechanical processing on the age-hardening behaviour of $6061 \mathrm{Al}-\mathrm{SiCp} \mathrm{MMC}$ produced by casting route. Scr.Metall.Mater. 1992; 27: 1139-1144.

17. Hong S H, Chung $\mathrm{K} \mathrm{H}$, Lee $\mathrm{C} \mathrm{H}$. Effects of hot extrusion parameters on the tensile properties and microstructures of SiCw-2124Al composites. Mater.Sci.Eng. 1996; 206A: 225-232.

18. Morimoto T, Yamaoka T, Lilholt H, Taya M. Second stage creep of SiC whisker/6061 aluminum composite at 573K. J.Eng.Mater.Tec. 1988; 110: 70-76.

19. González LM, Cumbrera FL, Sánchez-Bajo F, Pajares A. Measurement of fiber orientation in short-fiber composites, Acta Metall Mater 1994; 42: 689-694.

20. I.Bronshtein I, Semendiaev K, Mathematics handbook, MIR Moscu. 1993; 399-400. 


\section{Appendix}

The following relations allow to state equation [6] in terms of a complete first class elliptical integral, equation [7] by means of basic trigonometry:

$$
\begin{aligned}
& 1+\cos ^{2} \beta \cdot \operatorname{tg}^{2} \theta=1+\left(1-\sin ^{2} \beta\right) \cdot \operatorname{tg}^{2} \theta=1+\operatorname{tg}^{2} \theta-\sin ^{2} \beta \cdot \operatorname{tg}^{2} \theta= \\
& =\frac{\cos ^{2} \theta}{\cos ^{2} \theta}+\frac{\sin ^{2} \theta}{\cos ^{2} \theta}-\sin ^{2} \beta \cdot \frac{\sin ^{2} \theta}{\cos ^{2} \theta}=\frac{1}{\cos ^{2} \theta}-\frac{\sin ^{2} \beta \cdot \sin ^{2} \theta}{\cos ^{2} \theta}=
\end{aligned}
$$

Therefore:

$$
1+\cos ^{2} \beta \cdot \operatorname{tg}^{2} \theta=\frac{1}{\cos ^{2} \theta}\left(1-\sin ^{2} \beta \cdot \sin ^{2} \theta\right)
$$




\begin{tabular}{|c|c|c|c|c|}
\hline Material code & $\mathrm{T}_{\text {ext }}\left({ }^{\circ} \mathrm{C}\right)$ & $\mathrm{F}_{\mathrm{v}}(\%)$ & $a$ & $b$ \\
\hline $\mathrm{C} 32$ & 300 & 26 & 0.742 & 5.493 \\
\hline $\mathrm{C} 34$ & 359 & 25 & 0.972 & 7.762 \\
\hline $\mathrm{C} 38$ & 498 & 31 & 2.511 & 17.552 \\
\hline $\mathrm{C} 45$ & 532 & 31 & 1.635 & 10.841 \\
\hline
\end{tabular}

Table 1.- Extrusion temperature, $\mathrm{T}_{\mathrm{ext}}$, volume fraction of whiskers which are aligned with the extrusion axis, $F_{v}$ (the remaining fraction, $1-F_{v}$, are whiskers randomly oriented in the aluminum matrix), and the parameters $a$ and $b$ of the Gaussian function which determine the level of alignment of the population of oriented of whiskers of the $6061 \mathrm{Al}-15 \mathrm{vol} \% \mathrm{SiC}$ composites investigated (14). 


\section{List of Figures}

Figure 1.- Schematic view of the jutted out part of a fiber of length $l$ misaligned an angle $\theta$ with the symmetry axis. The corresponding projected length and projected angle on the metallographical plane ( $l^{\prime}$ and $\theta^{\prime}$, respectively) and the azimuth angle, $\beta$, are also shown.

Figure 2.- Variation of the real spatial length, $l$, of individual fibers as function of their angle $\theta$ with the symmetry axis for the case in which the projected length, $l^{\prime}$, is constant with the projected angle $\theta^{\prime}$. In this case, an analytical solution of equation [5] can be found.

Figure 3.- Inverse stereographic triangle (inverse pole figure of the extrusion axis) corresponding to the reinforcing phase of one of the $\mathrm{SiC}$ reinforcing 6061 Al composites investigated (C38). It is seen the trend of the whiskers axis $(<111>$ crystallographic direction) to align with the extrusion axis leading to a fiber texture on the reinforcing phase in these composites.

Figure 4. Scanning electron micrograph showing a longitudinal section of the composite C38, extruded at $498^{\circ} \mathrm{C}$, and the corresponding processed picture used to measure $l^{\prime}$ and $\theta^{\prime}$ (extrusion direction is the horizontal).

Figure 5.- Projected whisker length, $l^{\prime}$, (average) as a function of $\theta^{\prime}$, the projected angle with extrusion axis. Measurement dispersion is given at each angle.

Figure 6.- Real whisker length, $l$, and projected whisker length, $l^{\prime}$, as a function of the misorientation angle, $\theta$, with the symmetry (extrusion) axis for the four composites investigated. 


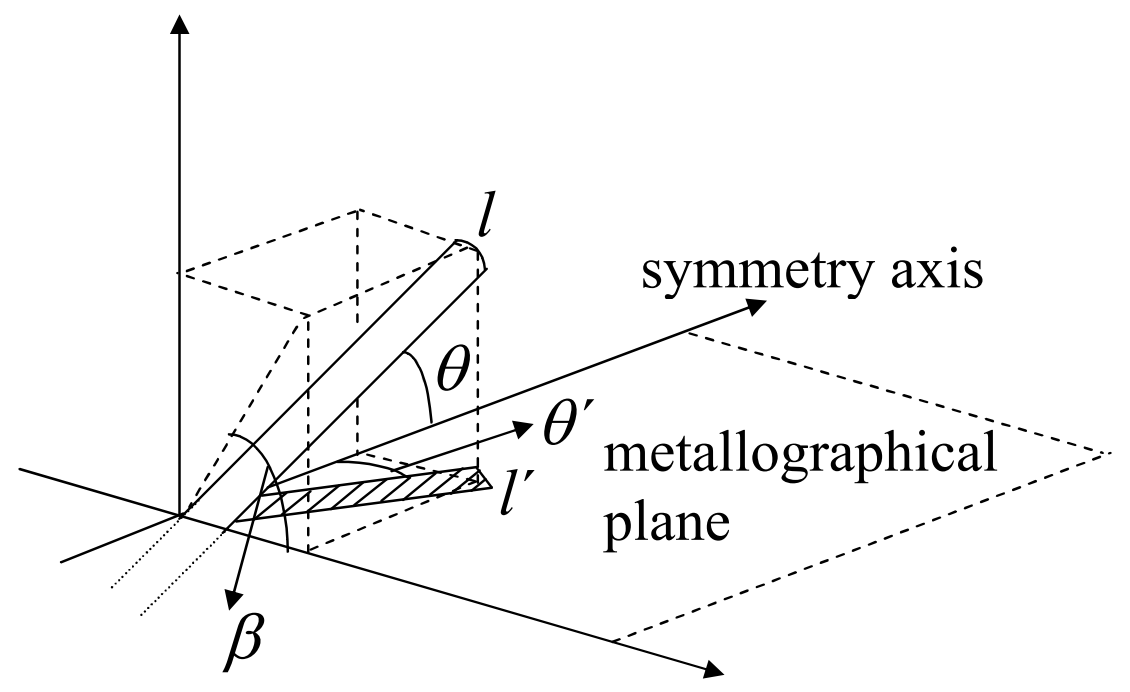

Figure 1. 


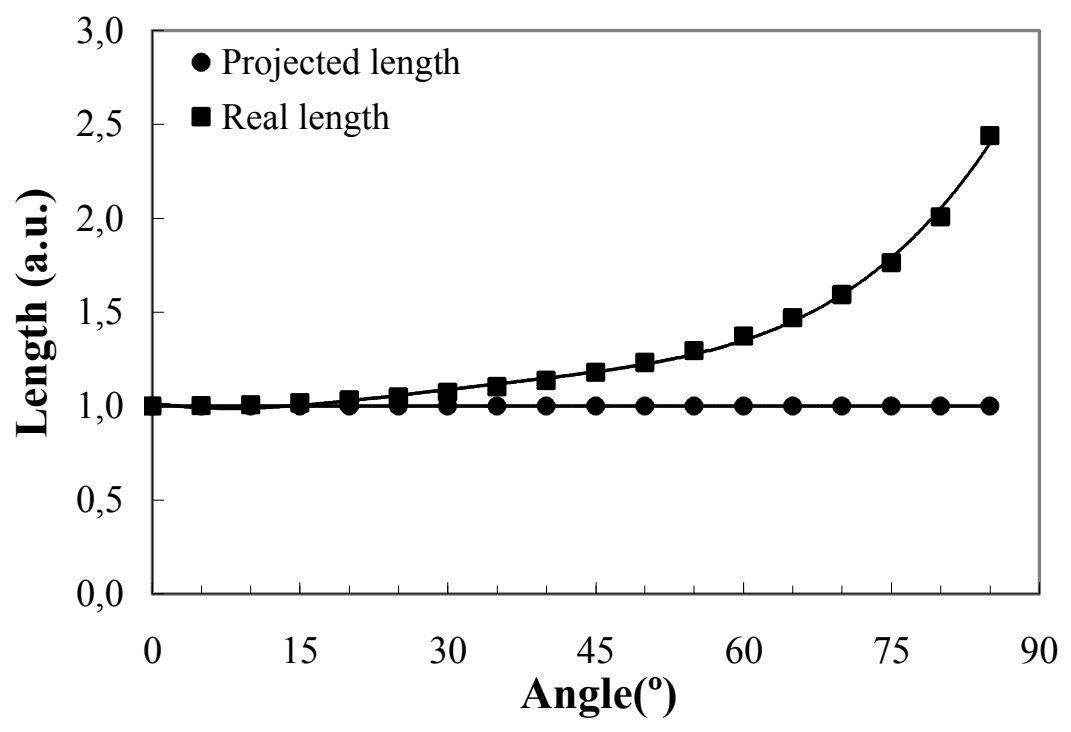

Figure 2. 


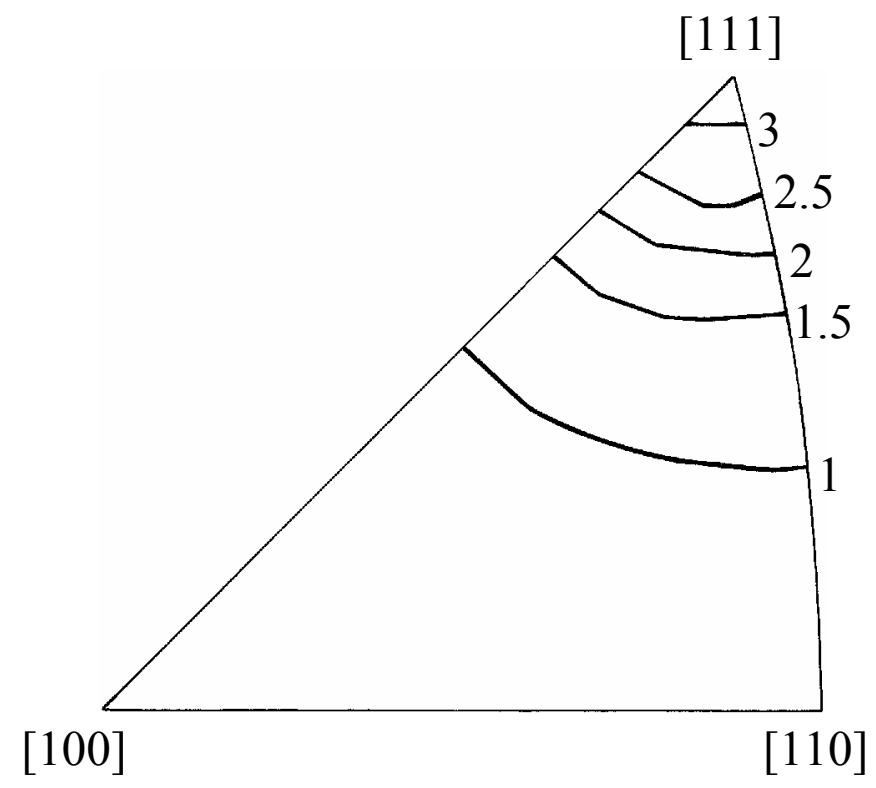

Figure 3. 


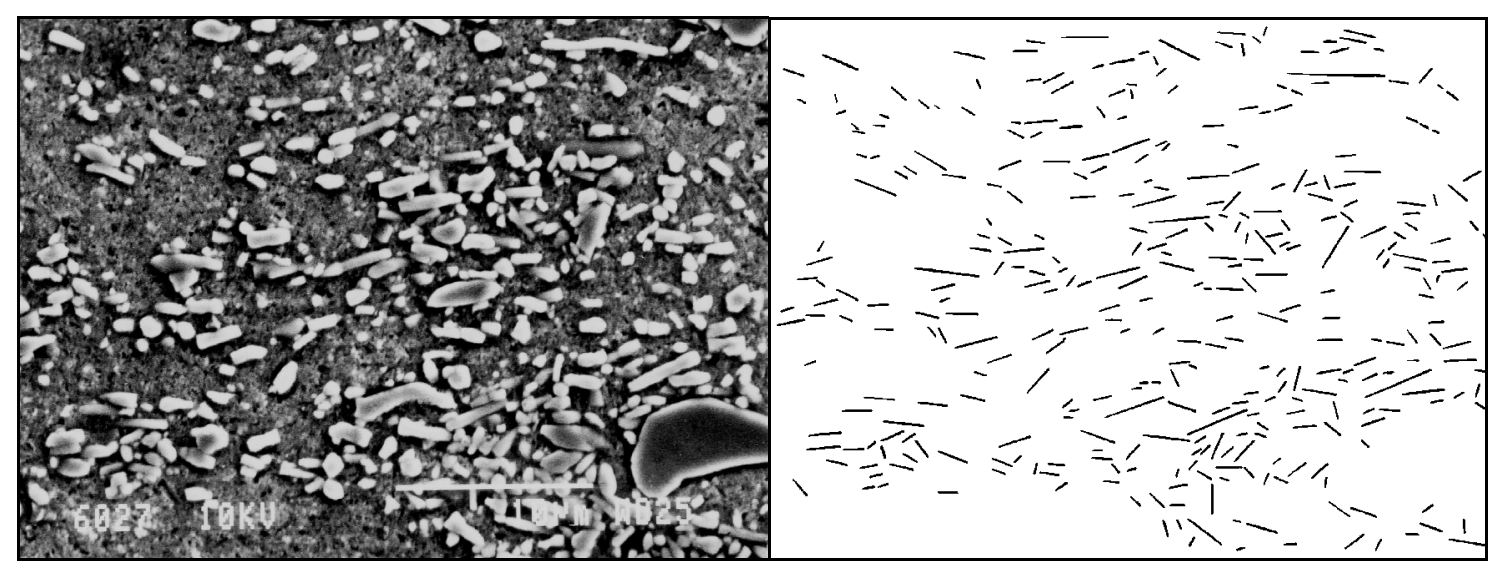

Figure 4. 

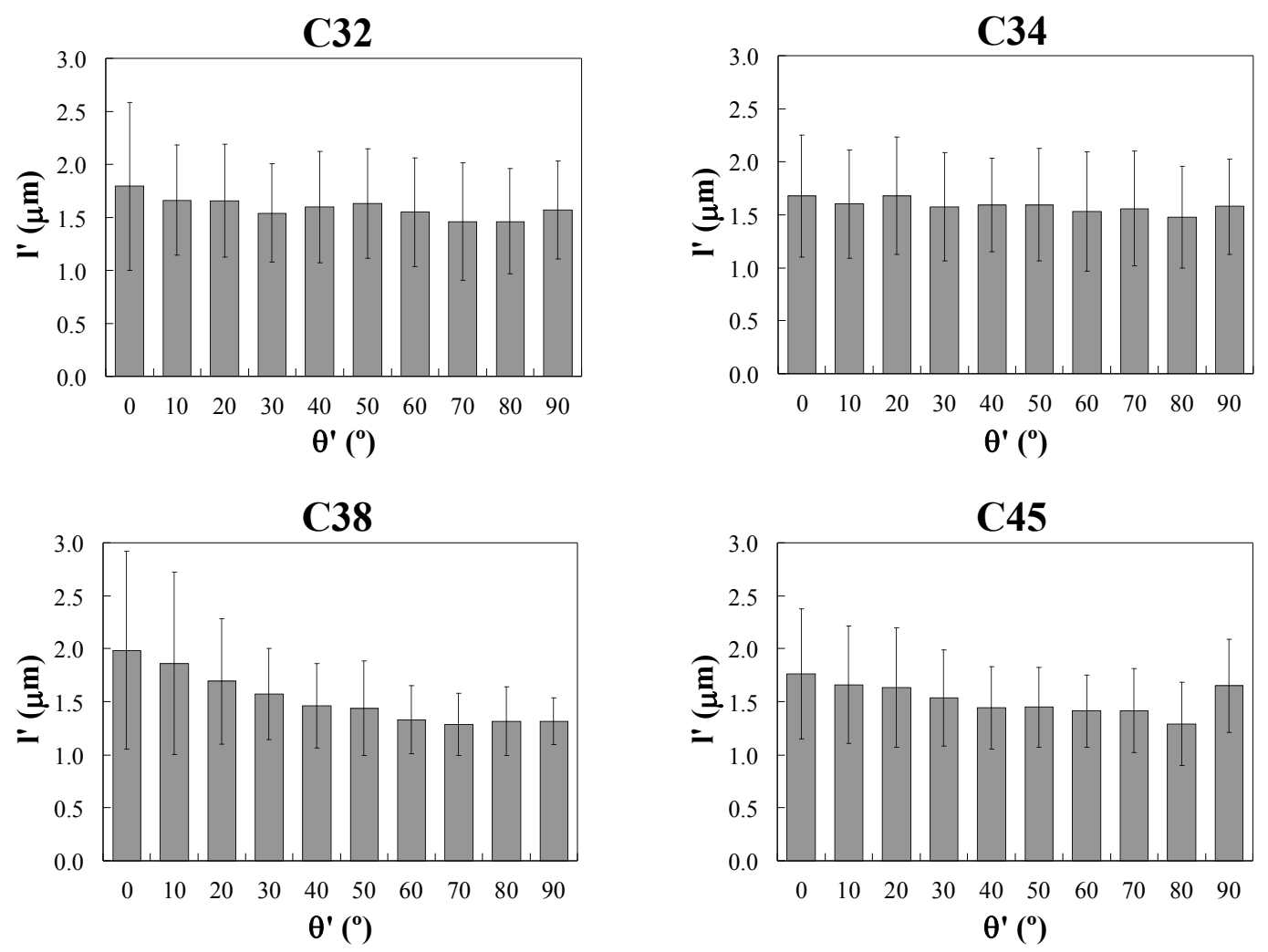

Figure 5. 

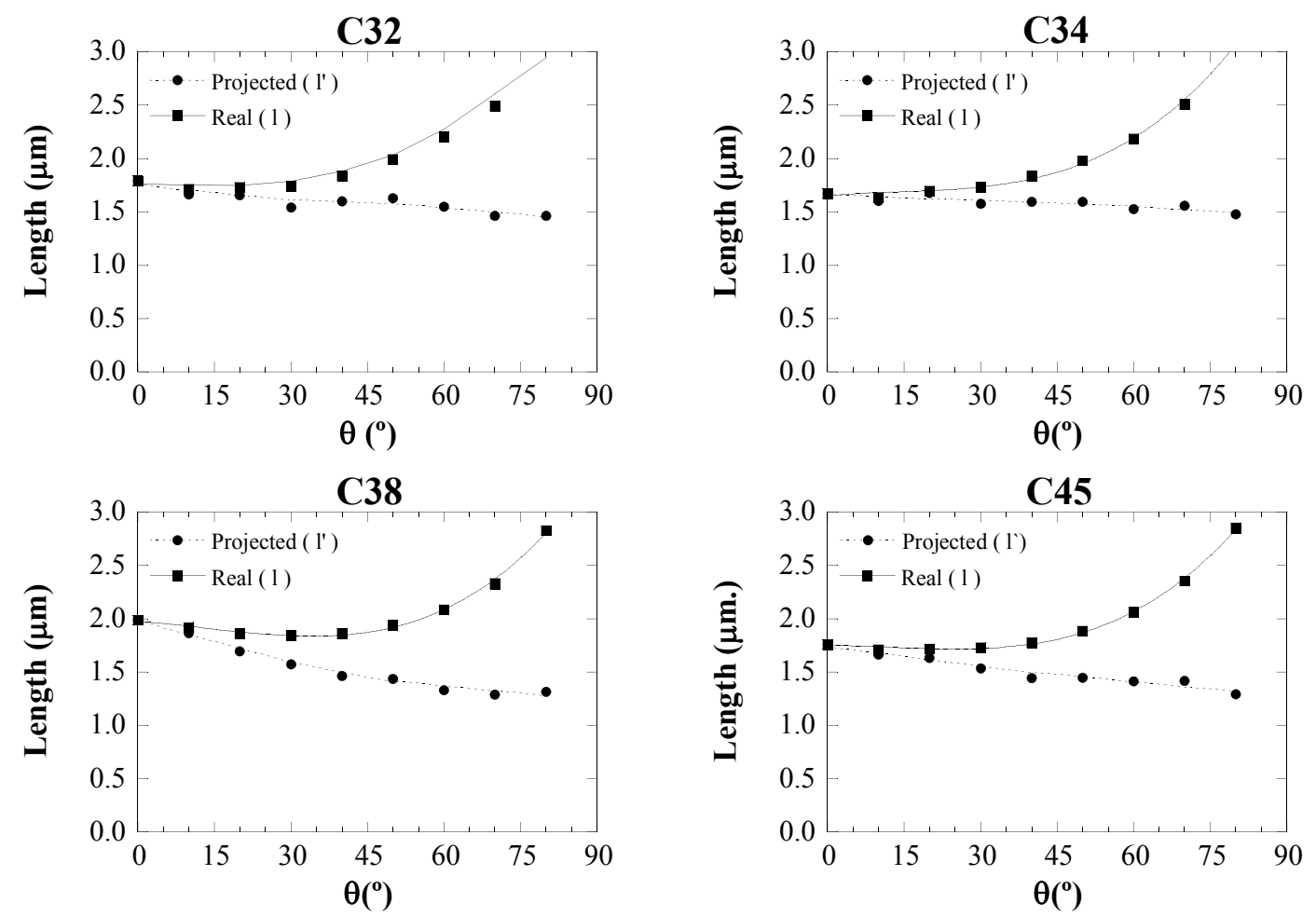

\section{Figure 6.}

